\title{
MULTIFRAGMENTATION IN REACTIONS OF 1-GEV PROTONS WITH IRON NUCLEI
}

\author{
D. MANCUSI ${ }^{*}$, A. BOUDARD ${ }^{1}$, R. J. CHARITY ${ }^{2}$, J. CUGNON, J.-C. DAVID ${ }^{1}$, S. LERAY ${ }^{1}$, A. KELIĆ-HEIL ${ }^{3}$, and M. \\ V. RICCIARDI ${ }^{3}$ \\ University of Liège, AGO Department, allée du 6 août 17, bât. B5, B-4000 Liège 1, Belgium \\ ${ }^{1} \mathrm{CEA}, \mathrm{SPhN}$, IRFU, Saclay, France \\ ${ }^{2}$ Department of Chemistry, Washington University, St. Louis, MO, U.S.A. \\ ${ }^{3}$ Reactions and Nuclear Astrophysics, GSI, Darmstadt, Germany \\ ${ }^{*}$ Corresponding author. E-mail : d.mancusi@ulg.ac.be
}

Received

Accepted for Publication

We use combinations of cascade and nuclear de-excitation models to assess the importance of the inclusion of a multifragmentation stage for the quantitative description of the $1-\mathrm{GeV} p+{ }^{56} \mathrm{Fe}$ spallation reaction. We discuss the sensitivity of several exclusive physical observables to the de-excitation mechanism in the attempt to identify which of them can be considered suitable probes for a multifragmentation stage. The calculations show that the inclusion of a multifragmentation stage is not necessary to construct an accurate description of the observed data.

KEYWORDS : multifragmentation, spallation, IMFs

\section{INTRODUCTION}

The role of multifragmentation $(\mathrm{MF})$ in spallation reactions is the subject of a long-standing discussion. It is generally agreed that highly-excited nuclear matter is spinodally unstable and that it should spontaneously break up into several fragments and unbound nucleons [1]. However, it is not clear whether and to what extent a MF model is actually necessary for a reliable quantitative description of spallation reactions around $1 \mathrm{GeV}$. Multifragmentation will eventually set in at higher projectile energy, but the 1$\mathrm{GeV}$ region, which is interesting for technical applications, remains an unsettled borderline case.

Not all nuclear de-excitation models include a MF stage; pure binary-decay models (coupled with a suitable intranuclear-cascade stage) have already proved able to reproduce accurately several observables, such as doubledifferential nucleon spectra or residue yields [2,3,4]. Hence, characteristic signatures of MF must be sought among other, more discriminating observables, such as correlations. The impact of a MF stage in the de-excitation chain can then be assessed by comparing calculation results with experimental data.

The goal of the present work is, firstly, to ascertain if multifragmentation needs to be postulated in order to construct an accurate description of several discriminating observables, such as longitudinal-velocity distributions or emission patterns of intermediate-mass fragments (IMFs); and secondly, to clarify to what extent such observables can exhibit characteristic signatures of multifragmentation. The focus of the paper is on the well-studied 1-GeV $p+{ }^{56} \mathrm{Fe}$ system [5, 6,7]. This reaction is expected to provide the optimal conditions for this study, since it can give rise to cascade remnants that are at the same time highly-excited and relatively massive.

\section{MODEL OVERVIEW}

The $1-\mathrm{GeV} p+{ }^{56} \mathrm{Fe}$ reaction was simulated by coupling the latest versions of the Liège Intranuclear Cascade model (INCL4 .5) [8] with the ABLA07 [9] and the GEMINI++ [10] de-excitation codes.

The INCL 4.5 model can be applied to collisions between nuclei and pions, nucleons or light nuclei of energy lower than a few GeV. The particle-nucleus collision is schematised as a sequence of binary collisions among the particles present in the system.; particles that are unstable over the time scale of the collision, notably $\Delta$ resonances, are allowed to decay. The nucleus is schematised as a square potential well whose radius depends on the nucleon momentum; thus, nucleons move on straight lines until they undergo a collision with another nucleon or until they reach the surface, where they escape if their total energy is positive and they manage to penetrate the Coulomb barrier. If the phase-space neighbourhood of the escaping nucleon is sufficiently populated, light clusters can be formed. The INCL 4. 5 model simulates a complete collision event, its output being the velocities of all the emitted particles. The characteristics of the remnant (its mass, charge, momentum, 
excitation energy and intrinsic angular momentum) are derived from the application of conservation laws and are passed to the chosen de-excitation code; the latter simulates the decay of the remnant into a nuclear-stable residue plus a number of nucleons, nuclei and/or gamma rays.

The ABLAQ7 model provides a rather complete description of nuclear de-excitation. It includes a MF module, an evaporation module and a fission module. Multifragmentation is the first possible de-excitation step and it is followed by a binary-decay phase, where compound nuclei can fission (if they are heavy enough) or evaporate nucleons, light nuclei and IMFs. The fission process is treated dynamically, using parametrisations of solutions of Fokker-Planck-type diffusion equations. Evaporation is described by the Weisskopf-Ewing formalism; conservation of angular momentum is approximately accounted for using a semi-empirical prescription. ABLAQ7's MF mechanism, albeit simple, provides at least a rough estimate the importance of this contribution. The MF module is triggered if the temperature of the remnant exceeds a given mass-dependent critical temperature, which is calculated following Natowitz et al.'s analysis [11]. The masses of the break-up fragments are then distributed according to semi-empirical power-law spectra [12].

The GEMINI++ model, on the other hand, does not include any MF mechanism but it contains a sophisticated model for the emission of IMFs. Emission of nucleons and nuclei up to $Z=3$ is treated in Hauser-Feshbach's evaporation framework; nuclei of charge $Z=4$ and above are assumed to be emitted in asymmetric fission-like processes, à la Moretto [13]. Symmetric fission is possible for heavy nuclei and it is described by a Bohr-Wheeler-type width.

\section{RESULTS}

Recent benchmark results [14], depicted in Fig. 11, show that the residue charge distributions predicted by the INCL4.5/ABLAQ7 and INCL4.5/GEMINI++ models are in good agreement with the experimental data [5,6,7]; this is a necessary condition to discuss the importance of multifragmentation for this reaction. The calculated cross sections are sums over the isotopes measured by Villagrasa et al. [5]. The INCL4.5/GEMINI++ prediction slightly overestimates the measured IMF yields, in the range $5 \lesssim A \lesssim 20$; this used not to be the case with older versions of the models [7].

The IMF cross sections are the most sensitive to MF. Since all the model calculations are in the same ballpark, we cannot draw any conclusions about the production mechanism from these data alone. It is nevertheless instructive to consider the contribution of ABLAO7's MF mechanism. This can be evaluated by switching off the MF module and comparing the predictions of the code, which are represented in Fig. 1 by the solid (MF allowed) and dashed (MF suppressed) blue lines. We remark that suppressing MF increases the IMF yields, which seems incompatible with the hypothesis that these cross sections are dominated by the MF mechanism. The solution to this puzzle will be given at the end of the following section.

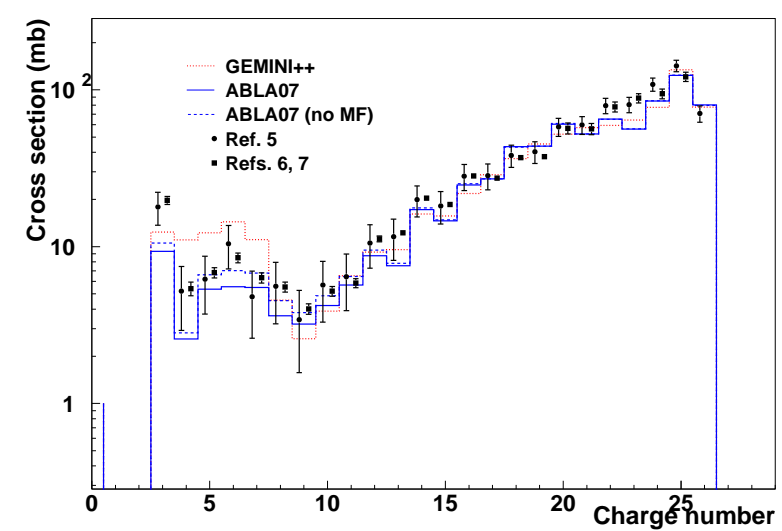

Figure 1. Residue charge distribution for the INCL4 . 5/ABLA07 (solid blue: with MF; dashed blue: without MF) and INCL4 . 5/GEMINI++ (solid red) models.

\subsection{Longitudinal-velocity distributions}

In order to provide an alternative interpretation, we therefore proceed to consider more discriminating observables. Napolitani et al. [6] measured longitudinal velocity distributions of several residues in reverse kinematics $\left(1 \mathrm{GeV} /\right.$ nucleon $\left.{ }^{56} \mathrm{Fe}+\mathrm{H}\right)$ using the FRagment Separator (FRS) at GSI, Darmstadt, Germany. The experimental setup had a limited acceptance in transverse momentum, which was accounted for in a simplified manner during the treatment of the simulation results. Light fragments showed a double-peaked velocity distribution, a manifestation of the existence of a Coulomb repulsion and, thus, of a binarydecay mechanism. As the mass of the fragment increases the distributions become single-humped, possibly indicating a transition towards another production mechanism, which was interpreted as multifragmentation.

The models considered in this work, however, provide a different explanation. Unfortunately, we cannot compare directly to the experimental data, since we do not have access to the experimental cuts. As Fig. 2 shows, however, the trend of the data is qualitatively reproduced by both models. The decomposition of the GEMINI++ distributions show that the lightest IMFs $\left({ }^{7} \mathrm{Li}\right)$ are mainly produced either as de-excitation products of a light asymmetric-fission partner (blue line) or as evaporation products (red line); both these components show double-peaked distributions. As the mass of the IMF increases, the single-humped contribution from de-excitation residues increases and masks the doublepeaked structure of the binary decays; moreover, even the asymmetric fission component is not as sharply peaked as in the case of ${ }^{7} \mathrm{Li}$.

The ABLA07 distributions (middle panels) also show a double-peaked evaporative component and are partly flattened out by the single-humped MF component, which amounts to $10-20 \%$ of the distribution. If one switches off MF, however, one remarks that the global shape of the distributions is not severely modified (bottom panels). The MF component is almost exactly compensated by an increase 


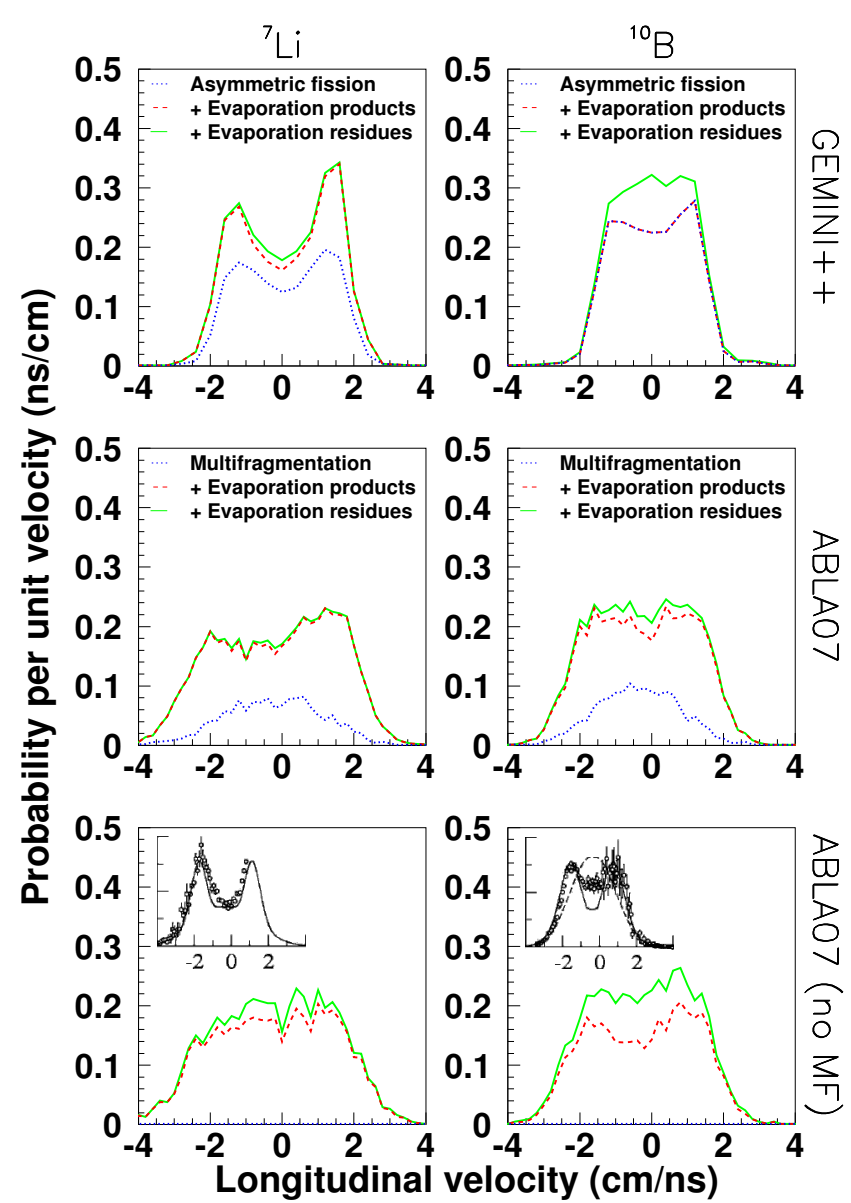

Figure 2. Longitudinal-velocity distributions for ${ }^{7} \mathrm{Li}$ (left column) and ${ }^{10} \mathrm{~B}$ (right column), as predicted by INCL4 . 5/GEMINI++ (first row) and by INCL4 . 5/ABLAQ7 (second row: with MF; third row: without MF). The residue velocity is calculated in the rest frame of the cascade remnant. Contributions from different production mechanisms are cumulated. The experimental results by Napolitani et al. [6] are shown in the insets in the bottom row (adapted).

in evaporation and in de-excitation residues. This is understandable if one considers that MF events are characterised by high excitation energy; thus, if multifragmentation is forbidden by hand, MF candidates will easily emit light nuclei and produce very light de-excitation residues. Incidentally, this explains why suppressing MF can lead to an increase of the inclusive cross section in Fig. 11 The almost miraculous compensation between multifragmentation and evaporation is probably characteristic of the $p+{ }^{56} \mathrm{Fe}$ system - it would be surprising if it occurred at other energies and for other targets, too.

\subsection{SPALADIN correlations}

Other discriminating observables are provided by the experiment performed with the SPALADIN setup at GSI [7]. Measurements of correlations between neutrons and charged particles are among the most selective tools to put
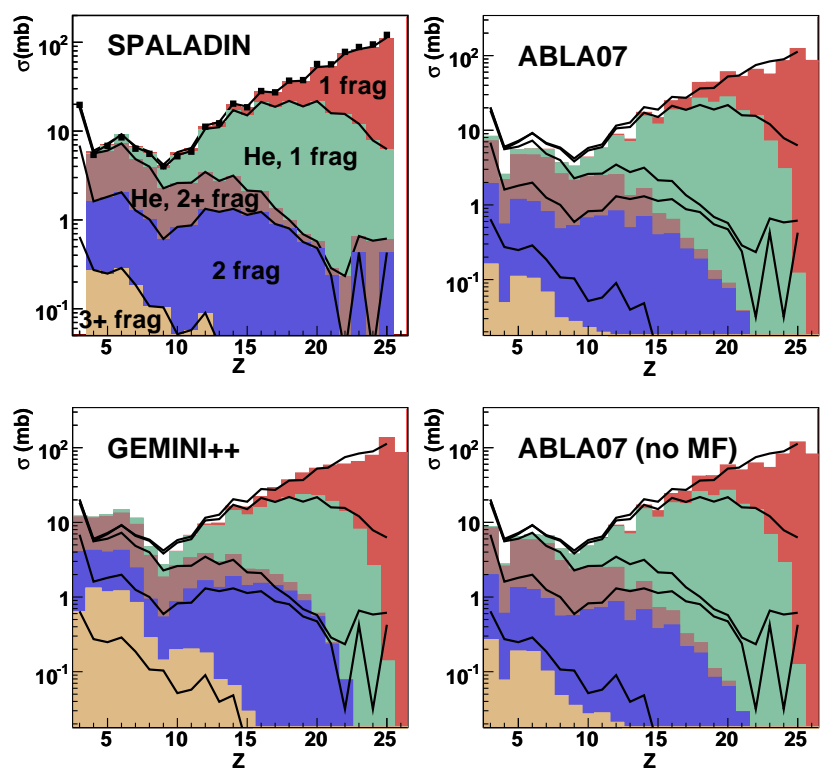

Figure 3. Charge distribution of residues, classified according to the presence of helium nuclei and the number of "fragments" $(Z \geq 3)$ observed in the event. In the second, third and fourth panel, the solid lines denote the experimental result and the coloured areas indicate the model prediction.

constraints on the de-excitation mechanism of compound nuclei. A similar analysis has already been performed with older versions of the models [7]. At that time, the INCL 4 . 2/GEMINI model was able to consistently reproduce all the considered observables. The old version of the ABLA model lacked a multifragmentation stage and a direct IMFemission mechanism, and was thus unable to describe the data. The new model versions provide different indications, as follows.

Fig. 3 shows the charge distribution of residues, classified according to the presence of helium nuclei and the number of "fragments" $(Z \geq 3)$ observed in the event. The calculation results were filtered with a GEANT4 transport calculation to be compared with the experimental data. The GEMINI++ model is more accurate for charges close to iron, while ABLA07 reproduces the classification of IMF events best. Suppressing MF slightly improves the agreement with the experimental data.

Finally, another test for the models is displayed in Fig. 4. Let $Z_{1}$ and $Z_{2}$ be the largest and the second-largest charge detected in a given event; Fig. 4 shows the cross section as a function of $Z_{1}-Z_{2}$, for three bins in neutron multiplicity plus helium multiplicity. The multiplicity bins can loosely be interpreted as bins in excitation energy. The GEMINI++ model is particularly good at reproducing the shape of the curves for low and medium multiplicity. At high multiplicity, the ABLAQ7 model does better and the partial failure of GEMINI++ is probably connected with the overestimation of IMF yields observed in Fig. 1. Multifragmentation, once again, does not seem to be decisive for the interpretation of the data. 


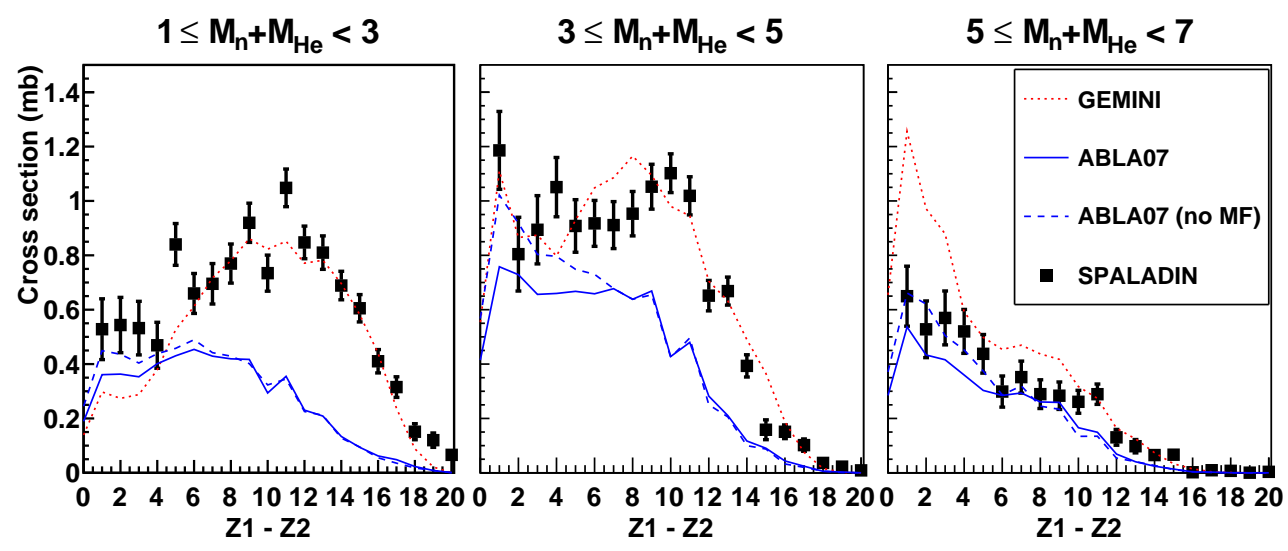

Figure 4. Cross section as a function of $Z_{1}-Z_{2}$, for three bins in neutron multiplicity plus helium multiplicity.

\section{CONCLUSIONS}

We have used the coupling of the INCL 4.5 cascade code with the ABLAQ7 and GEMINI++ nuclear de-excitation models to examine several discriminating observables connected with the $1-\mathrm{GeV} p+{ }^{56} \mathrm{Fe}$ spallation reaction, such as longitudinal-velocity distributions of residues and correlations among emitted neutrons and charged particles. We have found that most of the data can be consistently explained in a pure binary-decay framework, without invoking any multifragmentation mechanism, at least for this system.

\section{ACKNOWLEDGMENTS}

The authors express their gratitude to Thomas Gorbinet and Pekka Kaitaniemi for their help with the GEANT4 simulation of the SPALADIN setup.

\section{References}

[1] P. Chomaz, M. Colonna, and J. Randrup, "Nuclear spinodal fragmentation," Phys. Rep., vol. 389, pp. 263-440, January 2004.

[2] J.-J. Gaimard and K.-H. Schmidt, "A reexamination of the abrasion-ablation model for the description of the nuclear fragmentation reaction," Nucl. Phys. A, vol. 531, pp. 709745, September 1991.

[3] A. R. Junghans, M. de Jong, H.-G. Clerc, A. V. Ignatyuk, G. A. Kudyaev, and K.-H. Schmidt, "Projectile-fragment yields as a probe for the collective enhancement in the nuclear level density," Nucl. Phys. A, vol. 629, pp. 635-655, February 1998.

[4] A. Boudard, J. Cugnon, S. Leray, and C. Volant, "Intranuclear cascade model for a comprehensive description of spallation reaction data," Phys. Rev. C, vol. 66, p. 044615, October 2002.

[5] C. Villagrasa-Canton et al., "Spallation residues in the reaction ${ }^{56} \mathrm{Fe}+\mathrm{p}$ at $0.3 A, 0.5 A, 0.75 A, 1.0 A$, and $1.5 A \mathrm{GeV}$," Phys. Rev. $C$, vol. 75, p. 044603, April 2007.

[6] P. Napolitani, K.-H. Schmidt, A. S. Botvina, F. Rejmund, L. Tassan-Got, and C. Villagrasa, "High-resolution velocity measurements on fully identified light nuclides produced in
${ }^{56} \mathrm{Fe}+$ hydrogen and ${ }^{56} \mathrm{Fe}+$ titanium systems," Phys. Rev. C, vol. 70, p. 054607, November 2004.

[7] E. L. Gentil et al., "Coincidence measurement of residues and light particles in the reaction ${ }^{56} \mathrm{Fe}+p$ at $1 \mathrm{GeV}$ per nucleon with the spallation reactions setup SPALADIN," Phys. Rev. Lett., vol. 100, p. 022701, January 2008.

[8] J. Cugnon, A. Boudard, S. Leray, and D. Mancusi, "Results with INCL4," in Proceedings to the Satellite Meeting on Nuclear Spallation Reactions, International Topical Meeting on Nuclear Research Applications and Utilization of Accelerators, (Vienna, Austria), IAEA, May 2009. Submitted.

[9] A. Kelić, M. V. Ricciardi, and K.-H. Schmidt, "ABLA07towards a complete description of the decay channels of a nuclear system from spontaneous fission to multifragmentation," in Proceedings of the Joint ICTP-IAEA Advanced Workshop on Model Codes for Spallation Reactions (D. Filges, S. Leray, Y. Yariv, A. Mengoni, A. Stanculescu, and G. Mank, eds.), INDC(NDS)-530, pp. 181-222, IAEA, Trieste, Italy, $4^{\text {th }}-8^{\text {th }}$ February 2008.

[10] R. J. Charity, "GEMINI: a code to simulate the decay od a compound nucleus by a series of binary decays," in Filges et al. [9], pp. 139-148.

[11] J. B. Natowitz, R. Wada, K. Hagel, T. Keutgen, M. Murray, A. Makeev, L. Qin, P. Smith, and C. Hamilton, "Caloric curves and critical behavior in nuclei," Phys. Rev. C, vol. 63, p. 034618, March 2002.

[12] C. Sfienti et al., "Gross properties and isotopic phenomena in spectator fragmentation," Nucl. Phys. A, vol. 787, pp. 627632, May 2007.

[13] L. G. Moretto, "Statistical emission of large fragments: a general theoretical approach," Nucl. Phys. A, vol. 247, pp. 211-230, August 1975.

[14] S. Leray et al., "TBA," in Proceedings of the International Conference on Nuclear Data fr Science and Technology, (Jeju Island, Korea), Korean Nuclear Society and Korea Atomic Energy Research Institute, 26 ${ }^{\text {th }}-30^{\text {th }}$ April 2010. 